MURRAY AND TRETTEL, INCORPORATED

DENNIS W. TRETTEL

\section{Certified Consulting Meteorologist}

Founded 1946, Micro/Operational Forecasting • Media • Air Quality/

Meteorological/PSD Monitoring/Studies • Forensic Research

- Dispersion Modeling • Nuclear Emergency Support

600 First Bank Drive, Suite A

Palatine, IL 60067

$847-963-9000$

FAX: 847-963-0245

E-mail:mt@weathercommand.com

\section{NORTH AMERICAN WEATHER}

CONSULTANTS

DON A. GRIFFITH, PRESIDENT

\section{Certified Consulting Meteorologist}

Weather Modification • Air Quality Surveys \& Field Studies • Applied Research • Forensic Meteorology

8180 South Highland Dr., Suite B-2

Sandy, UT 84093

801-942-9005

FAX 801-942-9007

E-mail: nawc@nawcinc.com
WEATHER RESEARCH CENTER JOHN C. FREEMAN WEATHER MUSEUM JILL F. HASLING, DIRECTOR

\section{Certified Consulting Meteorologist}

Worldwide Weather \& Oceanographic Forecasting • Climatology

- Training • Expert Testimony • Research in Meteorology \&

Oceanography $\bullet$ Wave Spectra $\bullet$ Software Development $\bullet$ The WRC

Weather Museum

5104 Caroline St.

Houston, TX 77004

Phone: 713-529-3076

Fax: 713-528-3538

Website: www.wxresearch.com E-mail:WRC@wxresearch.org

\title{
ATMOSPHERIC SYSTEMS
}

\section{CORPORATION}

KENNETH H. UNDERWOOD, Ph.D.

\section{Certified Consulting Meteorologist}

Sodar Systems/Boundary Layer Studies • Meteorological Instruments and Systems/Meteorological Analysis • Wind Engineering Studies/Micrometeorology/Mixing Depth Studies - Acoustic Propagation/Noise Studies/Expert Testimony

24900 Anza Drive, Unit D

phone: 661-294-9621

Santa Clarita, CA 91355 fax: 661-294-9667 E-mail: ken@minisodar.com

TRC ENVIRONMENTAL CORP.

GALE F. HOFFNAGLE

DOUGLAS R. MURRAY

ALFRED M. KLAUSMANN

PIETRO A. CATIZONE

JOSEPH S. SCIRE

LLOYD L. SCHULMAN

\section{Certified Consulting Meteorologists}

Environmental Consulting \& Research • Applied Meteorology • Air

Quality and Meteorological Monitoring $\bullet$ Diffusion Modeling $\bullet$ Tracer

Studies • Air Toxics Monitoring • Expert Testimony

1-800-TRC-5601

Offices in major industrial centers throughout the United States
MCVEHIL-MONNETT ASSOCIATES, INC.

GEORGE E. McVEHIL, Ph.D.

KENDALL C. NECKER

\section{Certified Consulting Meteorologists}

Air Quality Analysis and Monitoring $\bullet$ Permitting $\bullet$ Dispersion Modeling • Air Toxics • Meteorological Analysis •Industrial Meteorology $\bullet$ Litigation Support $\bullet$ Expert Testimony
APPLIED METEOROLOGY, INC.

JOHN W. HATHORN

\section{Certified Consulting Meteorologist}

Meteorological Consulting \& Analysis • Air Quality Modeling \&

Monitoring • Site Selection \& Permitting • Environmental Data

Acquisition Systems \& Network with Remote-Control

9110 Weymouth Dr.

Houston, TX 77031-3034

713-995-5004 E-mail: amihou@ earthlink.net
SIMPSON WEATHER ASSOCIATES, INC.

M. GARSTANG, Ph.D.

R.H. SIMPSON, Ph.D. (retired)

G. D. EMMITT, Ph.D.

\section{Certified Consulting Meteorologists}

Environmental Risk Assessment • Air Quality Modeling/Monitoring

- Instrumentation Development/Deployment

- Lidar simulation/application

809 E. Jefferson St

434-979-3571

Charlottesville, VA 22902

FAX: 43

\section{INTERNATIONAL CENTER FOR THE SOLUTION OF ENVIRONMENTAL PROBLEMS \\ JOSEPH L. GOLDMAN, PH.D., TECHNICAL DIRECTOR}

\section{Certified Consulting Meteorologist}

Broad Scope Assessments by Interdisciplinary Experts Worldwide

- Specialize in Mesometeorological Variations - Climate Change

- Problems Posed, Solutions Implemented \& Demonstrated

509 North 7th Street

Alpine, TX 79830

\section{2-837-0157}

E-mail: icsep@airmail.net

\section{CLIMATOLOGICAL CONSULTING CORPORATION}

LEE E. BRANSCOME, Ph.D., President

DOUGLAS A. STEWART, Ph.D.

\section{Certified Consulting Meteorologists}

Forensic Meteorology • Weather Risk Analysis

- Climate Studies - Computer Modeling of the Atmosphere

7338 155th Place North

Palm Beach Gardens, FL 33418

www.ccc-weather.com
$561-744-4889$

FAX: 561-744-5098

Ibranscome@ccc-weather.com

Certified Consulting Meteorologist: The certification program of theAmerican Meteorological Society is aimed at fostering the establishment and maintenance of a high level of professional competency, and mature and ethical counsel, in the field of consulting meteorology. Requirements of knowledge, experience, and character are determined by a five-person board. Objectives of the program and application procedures are described in full detail in the August 200I Bulletin (pp. 1689-1694). 


\section{AEROCOMP \\ JOSEPH A. CATALANO}

\section{Certified Consulting Meteorologist}

Expert Testimony $\bullet$ Climatological Analysis •Industrial Meteorology \& Air Impact $\bullet$ Atmospheric Modeling $\bullet$ Wind \& Ice Loading $\bullet$ Data Management Software \& Services

P.O. Box 26109

Santa Ana, CA 92799-6109

E-mail: ccm299@aerocomp.com
WILKENS WEATHER TECHNOLOGIES, L. P. RICHARD B. WILKENS, PRESIDENT

\section{Certified Consulting Meteorologist}

Specialists in Offshore, Energy, and Industrial Forecasting Worldwide - Hindcast and Climatological Studies • Custom Weather Graphics and Information

2925 Briarpark, 7th Floor Houston, TX 77042-3715

713-430-7100 (Toll Free) 800-503-5811

E-mail:wwt@wilkensweather.com Web site: http://www.wilkensweather.com
FREESE-NOTIS WEATHER, INC. HARVEY FREESE, M.S.

CHARLES NOTIS, M.S.

\section{Certified Consulting Meteorologists}

Worldwide Forecasts for: Agricultural \& Energy Commodities - Construction • Media • Highway Departments • Forensic Meteorology $\bullet$ Internet Service Provider $\bullet$ Weather Products through Internet

2411 Grand Ave

Des Moines, IA 50312

Phone: $515-282-9310$

Fax: 515-282-6832

E-mail: hfreese@weather.net Internet: www.weather.net/fn/ams
AIR SCIENCE CONSULTANTS, INC. SKYWATCH WEATHER CENTER ${ }^{\circledR}$ ANTHONY J. SADAR, M.S.

\section{Certified Consulting Meteorologist}

Forecasting Services for Industry, Government and News Media • Air Quality Modeling •Impact and Climatological Studies • Forensic Meteorology • Environmental Assessments

347 Prestley Rd.

412-221-6000

Bridgeville, PA 15017 FAX: 412-221-3160

E-mail: airsci@ skywatchweather.com

WEATHERDATA SERVICES, INC.

an AccuWeather Company

MICHAEL R. SMITH, CEO/FOUNDER

STEPHEN PRYOR, MANAGER RESEARCH METEOROLOGY

\section{Certified Consulting Meteorologists}

- Premier Meteorological Consultants Serving All Industries

- Forensic Services • Forecast Services • Expert Testimony

- Exclusive Technology • Extensive Database • Comprehensive Studies

245 North Waco Ste. 310

Wichita, KS 67202

www.weatherdata.com
Phone: 316-265-9127 Fax: 316-265-1949 sales@weatherdata.com

ACCU WEATHER, INC.

ELLIOT ABRAMS, SENIOR VICE PRESIDENT

STEPHAN M. WISTAR, SENIOR METEOROLOGIST

\section{Certified Consulting Meteorologists}

Meteorological Consultants Serving Industry, Government and the Media • Forensic Services • Forecast Services • Expert Testimony - Complete Database - Applied Information Technologies

385 Science Park Road State College, PA 16803 www.AccuWeather.com
$814-235-8626$ Fax: 814-235-8769 E-mail: forensics@accuweather.com

\section{METEOROLOGICAL EVALUATION}

SERVICES, CO. INC. (MES)

PATRICK T BRENNAN, PRESIDENT

\section{Certified Consulting Meteorologist}

Air-Quality Consulting • Expert Testimony •Industrial Meteorology - Nuclear Licensing Studies - Weather Investigations for Legal and Insurance Firms

165 Broadway

E-mail: info@ mesamity.com

\section{TRINITY CONSULTANTS}

GEORGE J. SCHEWE, PRINCIPAL CONSULTANT ANTHONY J. SCHROEDER, SENIOR CONSULTANT

\section{Certified Consulting Meteorologists}

Air Quality Consulting $\bullet$ Regulatory Modeling $\bullet$ Meteorology/Climatology - Dispersion Modeling Courses Worldwide • BREEZE® Dispersion Modeling Software $\bullet$ Litigation Support

Covington, $\mathrm{KY}$ 859-341-8100

\section{gschewe@trinityconsultants.com} tschroeder@trinityconsultants.com

SKY FIRE PRODUCTIONS, INC.

WeatherVideoHD.tv

WALTER A. LYONS, Ph.D., President

\section{Certified Consulting Meteorologist}

Royalty Free Time Lapse Weather Footage (HD and SD) for producers and educators $\bullet$ Post-Production Services • Informal Science Education Programs • Online Educational DVD Sales

46050 Weld County Road 13

Ft. Collins, CO 80524

Phone: $970-416-8851$
www.Sky-Fire.tv www. WeatherVideoHD.tv Fax: $970-482-8627$

\section{EDWARD E. HINDMAN (Ward), Ph.D.}

\section{Certified Consulting Meteorologist}

Specializing in Meteorological Analyses, Education, and Expert Testimony
201-406-2184 EHindman@compuserve.com

www.trinityconsultants.com

Offices Nationwide 800-229-6655

For professional card rates, please apply to:

Executive Director,American Meteorological Society, 45 Beacon St., Boston, MA 021 08-3693 


\section{ANTHONY (ANDY) JOHNSON}

\section{Certified Consulting Meteorologist}

Expert Testimony - Weather Investigations for Legal and Insurance

Firms • Forensic Meteorology • Consultant since 1979

3912 West Dale Ave.

Tampa, FL 33609

E-mail: AndyCCM@aol.com
Phone: $813-870-9696$

FAX: 813-872-7202 $813-878-2929$

METEOROLOGICAL SOLUTIONS INC. GEORGE W. WILKERSON

DAN A. RISCH

\section{Certified Consulting Meteorologists}

Air Quality Modeling \& Development • Permitting • Ambient Monitoring • Calibrations \& Audits • Tracer Studies $\bullet$ Forecasting • Custom Software $\bullet$ Applied Meteorology $\bullet$ CALPUFF Modeling $\bullet$ Field Studies
2257 South 1100 East Suite 203

Salt Lake City, UT 84106

Website: www.metsolution.com
801-474-3826 801-474-0766 info@metsolution.com

\section{HOW THE WEATHERWORKS}

H. MICHAEL MOGIL, PRESIDENT

\section{Certified Consulting Meteorologist}

Specializing in forensic meteorology, expert testimony, and data analysis for legal and insurance matters; also educational design and weather-based training and educational courses, science writing and weather photography.
1104 6th Street South www.weatherworks.com hmmogil@weatherworks.com Naples, FL 34102
Cell: $240-426-2900$ Fax: 202-742-2806
Phone: 239-592-6636
AIR WEATHER \& SEA CONDITIONS, INC. JAY ROSENTHAL, PRESIDENT

\section{Certified Consulting Meteorologist}

Expert testimony and data analysis for legal and insurance matters

- Accident weather reconstruction - Satellite Interpretation - Air

Pollution Transport • Excellent Client References • Emergency Response

P. O. Box 512

Pacific Palisades, CA 90272

Phone: 818-645-8632 $310-454-7549$

FAX: 310-454-7569

Website: www.weatherman.org E-mail: AirWeather@aol.com

\section{CARL LARRY PEABODY}

\section{Certified Consulting Meteorologist}

Expert Testimony; Forensic Meteorology; Climatological Studies; Meteorological and Oceanographic Analysis; Legal and Insurance Weather Investigation; Outdoor Events Weather Observations and Forecasts; Freelance Writing-Magazine Weather Articles
11611 Caprock

San Antonio, TX 78230-2102 e-mail: Ipeabody@satx.rr.com

\section{INTEGRATED ENVIRONMENTAL DATA KATHLEEN E. MOORE, PH.D.}

\section{Certified Consulting Meteorologist}

Renewable Energy • Forest \& Agricultural Meteorology $\bullet$ Certified Forester $\bullet Q A / Q C$ Audits $\bullet$ Boundary Layer Studies $\bullet$ Sodar \& Radar Profiling Analyses $\bullet$ Remote Sensing Studies

255 Fuller Rd.

Albany, NY 12203

kemoore@iedat.com www.iedat.com (518) $956-7030$

\section{MAYACAMAS WEATHER CONSULTANTS}

JOHN P. MONTEVERDI, Ph.D., DIRECTOR

\section{Certified Consulting Meteorologist}

Forensic Meteorology • Climate Studies $\bullet$ Litigation Support • Expert Testimony • Operational Forecasts and Nowcasts

4425 View Street

Oakland, CA 94611
415-882-9898 Fax: 510-653-4320
E-mail: montever@comcast.net Website: www.mayacamaswx.com
WEATHER DECISION TECHNOLOGIES, INC. RICHARD L. CARPENTER, JR., Ph.D.

J. WILLIAM CONWAY

\section{Certified Consulting Meteorologists}

Radar Meteorology • Severe Weather Nowcasting and Analysis • Mesoscale and Microscale Numerical Modeling • Aviation Weather • Forensic Meteorology • Expert Testimony

3100 Monitor Ave., Suite 280

Norman, OK 73072

405-579-7675

www.wdtinc.com www. hailtrax.com info@wdtinc.com

\section{ATMOSPHERIC INFORMATION \\ SERVICES}

JEFFREY M. FREEDMAN, ESQ., Ph.D.

\section{Certified Consulting Meteorologist}

Applied Meteorological Research $\bullet$ Field Project Design and

Management $•$ Climatological Analysis • Environmental Assessments

- Hydrometeorological Studies • Boundary Layer Studies • Forensic

Investigations $\bullet$ Expert Testimony

255 Fuller Road

Suite 296

Albany, NY 12203
Phone: $518-437-8712$

E-mail:jmf@atmosinfo.com

\section{AECOM}

WILLIAM GROOT

\section{Certified Consulting Meteorologists}

Air Quality Modeling \& Development • Evaluation • Permitting • Data Analysis • Air Toxics • Acid Deposition • Ozone • Complex Terrain - CALPUFF Modeling • Expert Testimony

300 Baker Avenue, Suite 290

Concord, MA 01742

$978-371-4000$ FAX: 978-371-2468

Certified Consulting Meteorologist:The certification program of theAmerican Meteorological Society is aimed at fostering the establishment and maintenance of a high level of professional competency, and mature and ethical counsel, in the field of consulting meteorology. Requirements of knowledge, experience, and character are determined by a five-person board. Objectives of the program and application procedures are described in full detail in the August 200I Bulletin (pp. I689-1694). 


\section{PROFESSIONAL DIRECTORY}

SPACE RESERVED FOR CERTIFIED CONSULTING METEOROLOGISTS

WALSH ENVIRONMENTAL, LLC

ARTHUR P. MIZZI, Ph.D., J.D.

\section{Certified Consulting Meteorologist}

Atmospheric/Air Pollution Modeling and Analysis $\bullet$ Regulatory

Compliance Advising •Wind Energy Resource Assessments • Climate

Change Impact Assessment and Downscaling Studies • Environmental Consulting and Permitting • Expert Testimony

Phone: 303-903-5544

4888 Pearl East Circle, Suite 108 Boulder, CO 80301

E-mail: amizzi Website: www.walshenv.com
ERM

DAVID H. MINOTT

\section{Certified Consulting Meteorologist}

Air Dispersion Modeling • Air Toxics • Risk Assessment

- Environmental Permitting and Consulting • Global Warming

Offices Throughout the U.S. and Worldwide

617-646-7802 david.minott@erm.com

www.erm.com

\section{CLIMATE \& ATMOSPHERIC RESEARCH}

\section{ASSOCIATES, LLC (CARA)}

THOMAS W. TESCHE, Ph.D.

\section{Certified Consulting Meteorologist}

Regional Climate \& Chemical Transport Modeling $\bullet$ 8-hr Ozone \& $P M 2.5$ SIP Modeling • MM5 \& WRF Mesoscale Modeling • CAMx,

CMAQ, GEOS-Chem Applications $\bullet$ Emissions Inventory Development

- Snow and lce Research • Litigation \& Expert Testimony

\section{CLIMATE PHYSICS, LLC}

EDWIN X BERRY, Ph.D.

\section{Certified Consulting Meteorologist}

In a world of climate delusions

We bring you valid conclusions

439 Grand Ave., \#147

Bigfork, MT 59911

406-471-1464

ed@climatephysics.com 
GEOMET TECHNOLOGIES, INC. MARK J. STUNDER

Environmental Expert Systems, Artificial Intelligence • Air Pollution Analysis, Modeling \& Monitoring $\bullet$ Weather Risk Management, Climatological Studies $\bullet$ Research and Operations

20251 Century Blvd.

Germantown, MD 20874
$301-428-9898$
FLEETWEATHER, INC.

Since 1969

TORE H. JAKOBSEN, M.S., CO-OWNER

Forecasts for Weather-Sensitive Industry $\bullet$ Past Weather Research \& Expert Testimony • Global Ship-Guidance \& Surveillance • Weathercasting $•$ Automated Weather Databases \& Delivery Systems

The Fleetweather Building, 2566 Route 52 Hopewell Jct., NY 12533 845-226-8300

Fax: 845-226-1918 E-mail: info@fleetweather.com
CONNECTICUT WEATHER CENTER, INC. WILLIAM JACQUEMIN, CHIEF METEOROLOGIST, PRESIDENT

PROFESSIONAL SERVICES: Weather Forecasting for Media, Utilities, Industry, Ski Areas, Government, Educational Svcs, and Insurance/Lawyer Reports

18 Woodside Avenue

Danbury, CT 06810-7123

Web site: www.ctweather.com
Phone: 203-730-CTWX (2899)

Fax: 203-730-CTFX (2839)

E-mail: weatherlab

\section{FOX WEATHER}

ALAN FOX, DIRECTOR

Satellite Analyses • Remote Sensing Studies • Site Forecasts • Extended Outlooks • Quantitative Precipitation Forecasts, Product Delivery via Internet

726 13th Street, Suite B

$805-469-1368$

Fortuna, CA 95540

\section{R. M. YOUNG COMPANY}

\section{Meteorological Instruments since 1964}

Sensors to Measure: Wind Speed •Wind Direction • Peak Gusts • Temperature • Pressure • Relative Humidity • Precipitation

2801 Aero-Park Drive

Traverse City, Michigan 49686
231-946-3980

Fax: 231-946-4772 www.youngusa.com

\section{METEO CONSULT}

HARRY A. OTTEN, MANAGING DIRECTOR MAARTEN NOORT, DIRECTOR

Worldwide Forecasting $\bullet$ Media $•$ Marine (legally recognized in Dutch law) • Leading Experts in Winter Road Maintenance • Agriculture • Data $\bullet$ Software $\bullet$ Research and Consulting $\bullet$ Lightning Data

P.O. Box 617

6700 AP Wageningen

31317399800

The Netherlands

WEATHERBANK, INC.

STEVEN A. ROOT, CCM

President/CEO

MICHAEL R. ROOT Executive Vice President/CFO

Operational Forecasting • Broadcast Services • Forensic Studies • Real-Time and Historical Weather Databases • Satellite Transmission Network • Weather/Air Quality Monitoring and Climatological Studies

\section{More Than 35 Years of Providing Quality Service}

1015 Waterwood Parkway, Suite J

Edmond, Oklahoma 73034
405-359-0773

Fax: 405-341-0115 www.weatherbank.com

\section{SONALYSTS, INC.}

RIP COLEMAN

PATRICK KELLY

KATHY LUCAS JOSH ROVERO

wXstation ${ }^{\circledR}$ Weather Display and Analysis Software

Integrated display of worldwide satellite imagery, radar, lightning, GRIB, aircraft positions, and text weather from multiple sources. Software development, systems integration, training, installation, and maintenance.

215 Parkway North

P.O. Box 280

Waterford, CT 06385
Phone: $860-442-4355$

Fax: 860-447-8883

Web: www.sonalysts.com

\section{WEATHER CENTRAL INC.}

SCIENCE ENGINEERING ASSOCIATES, INC. LYLE E. LILIE, PRESIDENT

114 C Mansfield Hollow Rd.

P. O. Box 605

Mansfield Center, CT 06250-0605

E-mail: LyleL@scieng.com
Phone: 860-450-1717 Fax: 860-450-1707 Web site: www.scieng.com
Weather Central empowers broadcasters to attract and connect with viewers through highly local forecasts and severe weather coverage. Our solutions advance the business objectives of the broadcast, online, and print industries. Our clients dominate their markets with the best technology available.
5725 Tokay Boulevard

Madison, WI 53719

URL: www.weathercentral.tv
Phone: 608-274-5789

E-mail:sales@weathercentral.t
Fax: $608-278-2746$

\section{Win in Weather ... Lead in News ${ }^{\mathrm{TM}}$}




\section{A. H. GLENN AND ASSOCIATES SERVICES \\ CLAUDE V. PALLISTER III}

Consultants in Meteorology and Oceanography since 1946

New Orleans Lakefront Airport

P. O. Box 26337

New Orleans, LA 70186
504-241-2222 FAX 504-241-2224
D J GILE, INC.

METEOROLOGICAL \& AIR QUALITY CONSULTING

Meteorological \& Air Quality Monitoring • Turnkey Program

Development $\bullet$ Worldwide Services $\bullet$ Environmental Auditing •

Quality Assurance Program Development $\bullet$ Customized Data

Acquisition \& Software Programming $\bullet$ Data Management \&

Reporting

P. O. Box 706

Kennebunkport, ME 04046

Phone: 207-967-5286 Fax: 207-967-4107

Internet: www.djgile.com

ALTOSTRATUS, INC.

HAIDER TAHA, Ph.D., PRESIDENT

Meteorological modeling $\bullet$ Photochemical and dispersion modeling

- Regulatory modeling for ozone and PM • Fine-resolution urban

meteorological modeling $\bullet$ Aerometric data analysis $\bullet$ Weather

derivatives and applied meteorology

Ph: 925-228-1573

URL: www.altostratus.com

$$
\text { Fax: 925-228-8473 }
$$

E-mail: haider@altostratus.com 


\section{TABLES OF CONTENTS FOR AMS JOURNALS}

\section{Journal of the Atmospheric Sciences}

Vol. 66, No. 3, March 2009

\section{ARTICLES}

Internal Feedbacks from Monsoon-Midlatitude Interactions during Droughts in the Indian Summer Monsoon. R. Krishnan, Vinay Kumar, M. Sugi, and J. Yoshimura

Formation of Jets and Equatorial Superrotation on Jupiter

TAPIO SchneIder AND Junjun LiU

A Spectral Stochastic Kinetic Energy Backscatter Scheme and Its Impact on Flow-Dependent Predictability in the ECMWF Ensemble Prediction System

..J. Berner, G. J. Shutts, M. Leutbecher, and T. N. Palmer

Analysis of Convective Transport and Parameter Sensitivity in a Single Column Version of the Goddard Earth Observation System, Version 5, General Circulation Model

. L. E. Ott, J. Bacmeister, S. Pawson, K. Pickering, G. Stenchikov, M. Suarez, H. Huntrieser, M. Loewenstein, J. Lopez, and I. Xueref-Remy

Sensitivity of the Upper Mesosphere to the Lorenz Energy Cycle of the Troposphere.

ERICH BECKER

Storm-Relative Helicity Revealed from Polarimetric Radar Measurement

Matthew R. Kumjian and Alexander V. Ryzhkov

The Vertical Structure of Mesoscale Convective Vortices

. Christopher A. Davis and Thomas J. Galarneau Jr.

Life Cycles of Hurricane-Like Vorticity Rings

Eric A. Hendricks, Wayne H. Schubert,

Richard K. Taft, Huiqun Wang, and James P. Kossin

Turbulent Condensation of Droplets: Direct Simulation and a Stochastic Model

Roberto PaOli and Karim Shariff

How Well Does Water Activity Determine Homogeneous Ice Nucleation Temperature in Acqueous Sulfuric Acid and Ammonium Sulfate Droplets? BRIAN D. SWANSON

A Wind Tunnel Study on the Shape, Oscillation, and Internal Circulation of Large Raindrops with Sizes between 2.5 and $7.5 \mathrm{~mm}$

Miklós Szakáll, Karoline Diehl, Subir K. Mitra, and Stephan Borrmann

Dynamics of Prediction Errors under the Combined Effect of Initial Condition and Model Errors .......... C. Nicolis, Rui A. P. Perdigao, and S. Vannitsem

$553-578$

$579-601$

$603-626$

$627-646$

$647-666$

$667-685$

$686-704$

$705-722$

$723-740$

$741-754$

$755-765$

$766-778$ 


\section{Journal of Physical Oceanography}

Vol. 39, No. 3, March 2009

\section{ARTICLES}

Gravity Waves in a Horizontal Shear Flow. Part I: Growth Mechanisms in the Absence of Potential Vorticity Perturbations .......................................................... Nikolaos A. BAKAS AND BRIAN F. FARRELL

Gravity Waves in a Horizontal Shear Flow. Part II: Interaction between Gravity Waves and Potential Vorticity Perturbations .......................................................NIKolaos A. BAKAS AND BRIAN F. FARRELL

Northward Penetration of Antarctic Intermediate Water off Northwest Africa

F. Machín And J. L. Pelegrí

A Long-Lasting Mode Water Vortex in the Northeast Atlantic Ocean...

. Gilles Reverdin, Jean-Claude Gascard, Bernard Le Cann, Louis Prieur, Michel Assenbaum, and Pascale Lherminier

Calculating Energy Flux in Internal Solitary Waves with an Application to Reflectance.....

Kevin G. Lamb and Van T. Nguyen

Sea Surface Height Signals as Indicators for Oceanic Meridional Mass Transports...

..JoËl J.-M. Hirschi, Peter D. Killworth, Jeffrey R. Blundell, and David Cromwell

Time-Varying Across-Shelf Ekman Transport and Vertical Eddy Viscosity on the Inner Shelf.

Anthony R. Kirincich AND John A. Barth

Evolution of a Random Directional Wave and Freak Wave Occurrence...

Takui Waseda, Takeshi Kinoshita, and Hitoshi Tamura

The Surface Circulation of the Caribbean Sea and the Gulf of Mexico as Inferred from Satellite Altimetry Aida Alvera-Azcárate, Alexander Barth, AND ROBERT H. WeISBERG

Dynamics of the Wind-Driven Sea Level Variation around Antarctica

Kazuya Kusahara and Kay I. Ohshima

Transport in an Idealized Three-Gyre System with Application to the Adriatic Sea Irina I. Rypina, Michael G. Brown, and Huseyin KoçaK

Observations of Polarity Reversal in Shoaling Nonlinear Internal Waves ...

E. L. Shroyer, J. N. Moum, AND J. D. Nash

An Adjoint Sensitivity Analysis of the Southern California Current Circulation and Ecosystem

.. Andrew M. Moore, Hernan G. Arango, Emanuele Di Lorenzo, Arthur J. Miller, and Bruce D. Cornuelle

Observed Interannual Variability of the Florida Current: Wind Forcing and the North Atlantic Oscillation ..Pedro N. DiNezio, Lewis J. Gramer, . William E. Johns, Christopher S. Meinen, and Molly O. Baringer

Numerical Study on the Variability of Mixed Layer Temperature in the North Pacific. Shin'ichiro Kako and Masahisa Kubota

The South China Sea Throughflow Retrieved from Climatological Data

. Max Yaremchuk, Julian McCreary Jr., Zuojun Yu, and Ryo Furue Observations of Ekman Currents in the Southern Ocean.

Yueng-Djern Lenn and Teresa K. Chereskin

Observations of the Transition Layer T. M. Shaun Johnston and Daniel L. Rudnick

$481-496$

$497-511$

$512-535$

$536-558$

$559-580$

$581-601$

$602-620$

$621-639$

$640-657$

$658-674$

675-690

691-701

$702-720$

$721-736$

$737-752$

$753-767$

$768-779$

$780-796$

\section{NOTES AND CORRESPONDENCE}

Impact of Horizontal Diffusion on the Nonlinear Stability of Thermohaline Circulation in a Modified Box Model.. XiaOgang Wu and Mu Mu 